\title{
An investigation into the effects of E-cigarette aerosols using a physiologically relevant in-vitro model
}

\author{
Pranav Vasanthi Bathrinarayanan ${ }^{1}$, Lindsay J. Marshall ${ }^{2}$, James E.P. Brown ${ }^{2}$, Laura J. Leslie ${ }^{1}$ \\ 1-School of Engineering and Applied Science, Aston University, Birmingham, B4 7ET, UK \\ 2-School of Life and Health Sciences, Aston University, Birmingham, B4 7ET, UK
}

Although widely popular as smoking cessation tools, limited information is available on the health effects of E-cigarette (EC) use. An urgent requirement of EC research is a standard testing method to investigate possible adverse effects. The present study aims to assess EC cytotoxicity using an invitro multicellular human airways model.

Human bronchial epithelial cells (CALU3) and pulmonary fibroblasts (MRC5) were co-cultured on permeable membranes for 11-14 days at air-liquid interface. A bespoke smoking machine was used to deliver air, whole cigarette smoke (WCS) or EC vapour (ECV) to the airways model under standard ISO:3308 conditions for $7 \mathrm{~m}$. Considering the prolonged vaping habits of EC users compared to cigarette smoking, ECV exposure was additionally investigated at $1 \mathrm{~h}, 2 \mathrm{~h}, 3 \mathrm{~h}, 4.5 \mathrm{~h}$ and $6 \mathrm{~h}$ time points.

$24 \mathrm{~h}$ post exposure, XTT cell viability analysis showed that while WCS had the expected detrimental impact on cell viability, air exposure had no effect at any time point. Interestingly, a steady decrease in the viability of ECV exposed cells was observed at times greater than $2 \mathrm{~h}$. Viability was $61.31 \pm$ $5.75 \%$ control, $51.11 \pm 5.56 \%$ control and $42.10 \pm 2.69 \%$ control after $3 \mathrm{~h}, 4.5 \mathrm{~h}$ and $6 \mathrm{~h}$ respectively. Furthermore, ELISA analysis of supernatants revealed an increase in IL-6/IL-8 pro-inflammatory cytokines at $3 \mathrm{~h}$ post ECV exposure, despite the increased cell death.

Results indicate that extended EC exposure ( $\geq 3 \mathrm{~h}$ ) under these conditions has a detrimental impact on cell viability and leads to exaggerated cytokine production in the airways model. 